\title{
Searches for invisible Higgs boson decays with ATLAS and CMS
}

\author{
P. Calfayan*, on behalf of the ATLAS and CMS Collaborations \\ Ludwig-Maximilians-Univesität München \\ Am Coulombwall 1, D-85748 Garching, Germany \\ E-mail: philippe.calfayanephysik.uni-muenchen.de
}

The ATLAS and CMS experiments carried out searches for invisible decays of the Higgs boson. The analyses have been performed in final states with missing energy in the transverse plane of the detector, in the case of typical signatures where the Higgs boson is produced in association with an electroweak vector boson or via gluon-gluon or vector boson fusion. Upper bounds on the production cross section of the Higgs boson times its invisible branching fraction have been derived depending on the assumed mass of the Higgs boson. Additional interpretations have been considered in the context of Higgs-portal models, which resulted in constraints on the production of Dark Matter candidates. The searches are based on the complete datasets from the Run-1 of the Large Hadron Collider, which includes proton-proton collision at a center-of-mass energy of $\sqrt{s}=7$ or $8 \mathrm{TeV}$, with up to $20 \mathrm{fb}^{-1}$ of integrated luminosity.

The European Physical Society Conference on High Energy Physics

22-29 July 2015

Vienna, Austria

${ }^{*}$ Speaker. 


\section{Introduction}

To complete the study of the properties of the Higgs boson, it is essential to search for its potential invisible decay modes. By constraining the invisible branching fraction of the Higgs boson, it is possible to probe deviations from the Standard Model (SM). In addition, limits on $\sigma \times$ $\mathrm{BR}(H \rightarrow$ invisible $)$, the production cross section of the Higgs boson times its invisible branching fraction, can also be set in the case of hypothetical Higgs bosons with masses $\mathrm{m}_{H}$ differing from the prediction of the SM. In Higgs-portal models [1, 2], the SM is connected to the dark sector via the Higgs boson, so that new couplings between the Higgs boson and Dark Matter (DM) candidates can be investigated.

In the following, invisible decays of the Higgs boson are examined, assuming it is produced in association with a $Z$ or a $W$ boson ( $Z H$ or $W H$ channels) or via gluon-gluon or vector boson fusion (VBF). The analyses of multiple final states are reported in this document, and upper bounds on $\sigma \times \mathrm{BR}(H \rightarrow$ invisible $)$ are derived. If not mentioned explicitly, all limits are computed at the $95 \%$ confidence level.

\section{Search in the mono-jet final state}

The ATLAS experiment carried out a search for new physics in the final state consisting of a high energy jet plus large missing transverse energy $\left(E_{\mathrm{T}}^{\text {miss }}\right)$ [3]. The analysis relies on $20 \mathrm{fb}^{-1}$ of data at $\sqrt{s}=8 \mathrm{TeV}$, and includes an interpretation assuming the invisible decay of the Higgs boson.

While the selection of the signal does not exclude events based on the number of jets, most of the surviving events have the mono-jet signature, and the search is particularly sensitive to the Higgs boson production via gluon-gluon fusion. The multi-jet background is suppressed by vetoing events where a jet points in the direction of the $E_{\mathrm{T}}^{\text {miss }}$, and events with a leptonic $W$ decay are reduced by vetoing on events containing an electron or a muon. The main remaining background from the SM is in the $Z$ boson production in association with jets, followed by its decay to neutrinos. It is determined from data control regions with leptonic $W$ or $Z$ decays. These are corrected by simulation to account for the acceptance of the control region cuts and the difference of phase space, branching fraction, and cross section between the control and signal processes.

By interpreting the results with a simple counting experiment, upper limits on $\sigma \times \mathrm{BR}(H \rightarrow$ invisible) are derived as function of $\mathrm{m}_{H}$. Figure 1, which provides these limits, shows that the sensitivity of the search increases for higher $\mathrm{m}_{H}$. Considering a SM Higgs boson at a mass of $125 \mathrm{GeV}$ and with a production cross section $\sigma_{\mathrm{SM}}$, the observed and expected upper bound on $\sigma \times \mathrm{BR}(H \rightarrow$ invisible $) / \sigma_{\mathrm{SM}}$ is 1.59 and 1.91 , respectively.

\section{Search in the mono-jet $/ W / Z$ final states}

The CMS experiment recently performed a search for new physics in events with a jet or a vector boson $\mathrm{V}$ ( $W$ or $Z$ ) balancing $E_{\mathrm{T}}^{\text {miss }}$ [4], using $20 \mathrm{fb}^{-1}$ of data at $\sqrt{s}=8 \mathrm{TeV}$.

The event selection requires large $E_{\mathrm{T}}^{\text {miss }}$, one or more jets, and no isolated lepton or photon. Three event categories are distinguished, depending on the nature of the particle balancing the 
$E_{\mathrm{T}}^{\text {miss }}$. Events with an unresolved (boosted) V-tag are selected by clustering a high energy jet using the Cambridge-Aachen algorithm [5] with a large distance parameter $R=0.8$, requiring the jet mass to be close to that of a W or $\mathrm{Z}$ boson. By contrast, resolved V-tagged events are selected by identifying a pair of high energy jets with the smaller distance parameter $R=0.5$, and in addition using a multivariate V-tagger. It is worth mentioning that resolved V-tagged events including a b-quark are discarded, in order to keep the orthogonality with the CMS search described in Section 5 [7]. Finally, events with no V-tag but a high energy jet are categorized as mono-jet events. The main backgrounds are the $W$ and $Z$ boson productions in association with jets. The $W$ plus jets background is estimated from a control region defined by inverting the veto on leptons, and the $Z$ to two neutrinos background is determined from a $Z$ to leptons control region. The limited statistics of the latter at high transverse momentum $\left(p_{\mathrm{T}}\right)$ of the boson is compensated by considering an additional control region enriched in photon plus jets events. The background is determined via a likelihood fit in the control regions plus the signal region for all three event categories.

Upper bounds on $\sigma \times \mathrm{BR}(H \rightarrow$ invisible $) / \sigma_{\mathrm{SM}}$ are calculated as function of $\mathrm{m}_{H}$ via a shape experiment based on $E_{\mathrm{T}}^{\text {miss }}$. The limits obtained by combining all three event categories are illustrated in Figure 2. In the case where $\mathrm{m}_{H}=125 \mathrm{GeV}$, the observed and expected upper limits on $\mathrm{BR}(H \rightarrow$ invisible $)$ are 0.53 and 0.62 , respectively.

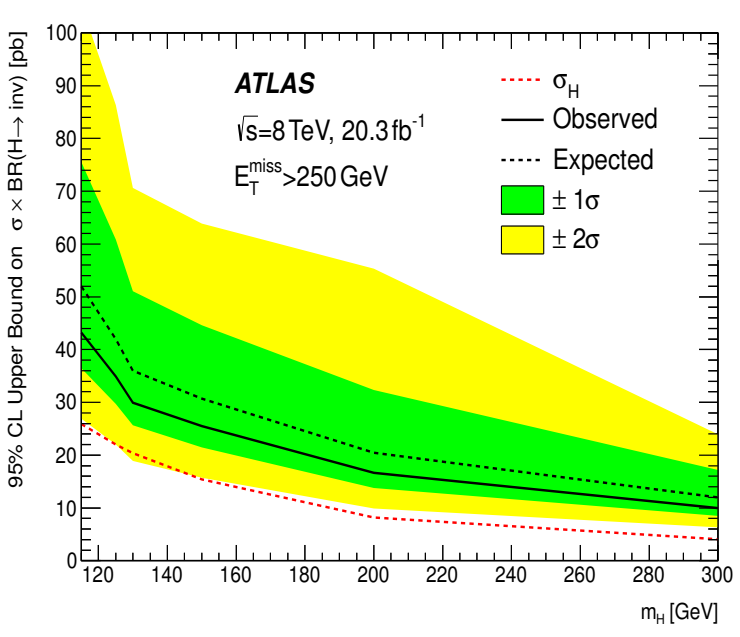

Figure 1: ATLAS mono-jet search [3]. Upper limits on the production cross section of the Higgs boson times its invisible branching fraction as function of the assumed mass of the Higgs boson.

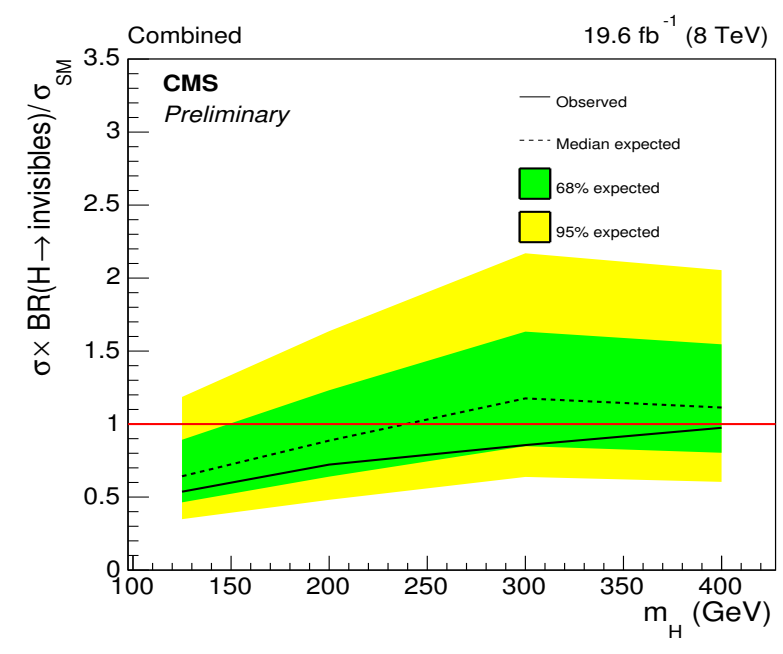

Figure 2: CMS mono-jet/W/Z search [4]. Upper bounds on the branching fraction of the Higgs boson decaying to invisible particles times the ratio of the Higgs boson cross section over that of the SM Higgs boson. Limits are shown as function of the assumed mass of the Higgs boson.

\section{Search in the $Z(\rightarrow \ell \ell) H$ channel}

Both the ATLAS and CMS experiments carried out a search for an invisible Higgs boson in events with the signature consisting of a $Z$ boson decaying to electrons or muons, and a Higgs boson decaying to invisible particles. The results in References $[6,7]$ were based on datasets from 5 to $20 \mathrm{fb}^{-1}$ of collisions at $\sqrt{s}=7$ or $8 \mathrm{TeV}$. 
To define the signal region, events with a large $E_{\mathrm{T}}^{\text {miss }}$ and containing two leptons with an invariant mass consistent with the $Z$ mass are kept. The specific topology of a $Z$ boson balancing a boosted invisible Higgs boson is enhanced by optimized requirements on the azimuthal angle difference between the transverse momentum of the di-lepton system $\left(p_{T}^{\ell \ell}\right)$ and the $E_{\mathrm{T}}^{\text {miss }}$, the fractional $p_{\mathrm{T}}$ difference between $p_{T}^{\ell \ell}$ and the $E_{\mathrm{T}}^{\text {miss }}$, and the azimuthal angle difference between the two leptons $(\Delta \Phi(\ell \ell))$. The main backgrounds are the $Z Z$ and $W Z$ di-boson productions, which are determined by simulation and normalized to a next-to-leading order production cross section. Besides, backgrounds with a pair of leptons not originating from a resonant decay, such as the $W W$, $t \bar{t}, W t$, or $Z \rightarrow \tau \tau$ productions, are estimated from data by considering a control region based on events including both an electron and a muon.

To compute the upper bounds on $\mathrm{BR}(H \rightarrow$ invisible $)$, ATLAS considers a maximum likelihood fit to the $E_{\mathrm{T}}^{\text {miss }}$ distribution, while CMS performs it to the two-dimensional distribution constructed from $\Delta \Phi(\ell \ell)$ and $\mathrm{m}_{T}$, the reconstructed mass of the di-lepton- $E_{\mathrm{T}}^{\text {miss }}$ system. In the case of the $7 \mathrm{TeV}$ analysis, CMS only considers the $\mathrm{m}_{T}$ distribution due to lower statistics. Under the hypothesis of a SM Higgs boson with $\mathrm{m}_{H}=125 \mathrm{GeV}$, the ATLAS observed and expected upper limits on $\mathrm{BR}(H \rightarrow$ invisible $)$ are 0.75 and 0.62 , respectively. The corresponding CMS observed and expected upper bounds amount to 0.83 and 0.86 , respectively. Figure 3 provides the ATLAS upper limits on $\sigma \times \operatorname{BR}(H \rightarrow$ invisible $)$ as function of $\mathrm{m}_{H}$.

\section{Search in the $Z(\rightarrow b \bar{b}) H$ channel}

The search for an invisible Higgs boson produced in association with a $Z$ boson decaying to a pair of $b$-quarks has been published by the CMS experiment [7]. The results are based on $20 \mathrm{fb}^{-1}$ of proton-proton collisions at $\sqrt{s}=8 \mathrm{TeV}$.

The signal region is enhanced in events with a boosted Higgs boson by requiring a large azimuthal angle difference between the $Z$ and the Higgs boson, and by distinguishing between three regions of $E_{\mathrm{T}}^{\text {miss }}$. In addition, events are required to include two high- $p_{\mathrm{T}} b$-tagged central jets, as well as to pass a minimum requirement on the $p_{\mathrm{T}}$ of the di-jet system. Events with a di-jet invariant mass exceeding $250 \mathrm{GeV}$, or with isolated leptons, are discarded. A final discrimination between signal and background is achieved via a boosted decision tree (BDT).

Limits on $\sigma \times \mathrm{BR}(H \rightarrow$ invisible $)$ are obtained with a $\mathrm{CL}_{s}$ procedure by combining the BDT output from the three regions of $E_{\mathrm{T}}^{\text {miss }}$. Assuming a SM Higgs boson with $\mathrm{m}_{H}=125 \mathrm{GeV}$, the observed (expected) upper bound on $\sigma \times \mathrm{BR}(H \rightarrow$ invisible $) / \sigma_{\mathrm{SM}}$ amounts to 1.82 (1.99). However, when combining this analysis with the $Z(\rightarrow \ell \ell) H$ search described in Section 4 [7], CMS improves this upper limit down to 0.81 (0.83). Figure 4 shows the upper bounds on $\sigma \times \mathrm{BR}(H \rightarrow$ invisible $)$ as function of $\mathrm{m}_{H}$ in the case of the combination of the $Z(\rightarrow b \bar{b}) H$ and $Z(\rightarrow \ell \ell) H$ searches.

\section{Search in the $W / Z(\rightarrow j j) H$ channel}

ATLAS has recently published a search for invisible decays of a Higgs boson produced in association with a vector boson ( $W$ or $Z$ ) decaying hadronically. The analysis relies on on $20 \mathrm{fb}^{-1}$ of data at $\sqrt{s}=8 \mathrm{TeV}[8]$. 


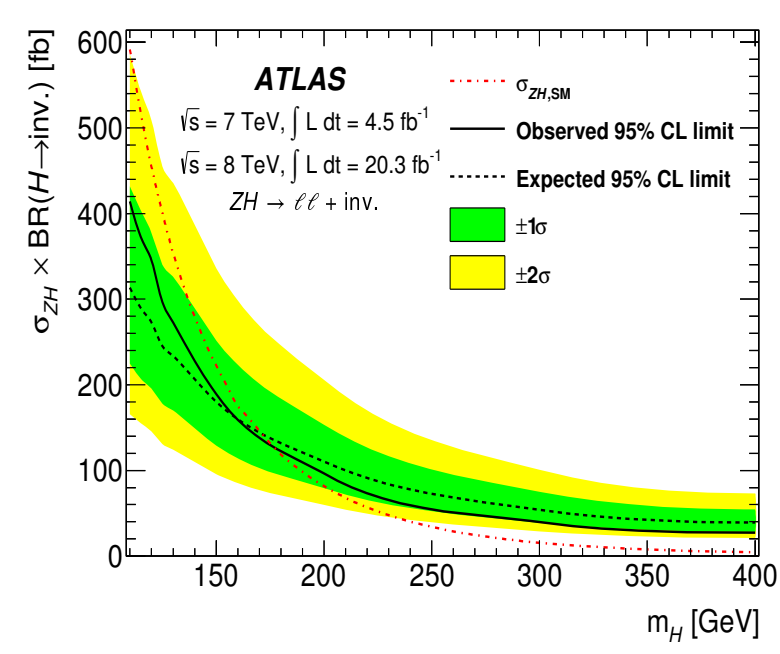

Figure 3: ATLAS mono- $Z(\rightarrow \ell \ell) H$ search [6]. Upper bounds on the production cross section of the Higgs boson times its invisible branching fraction as function of the assumed mass of the Higgs boson.

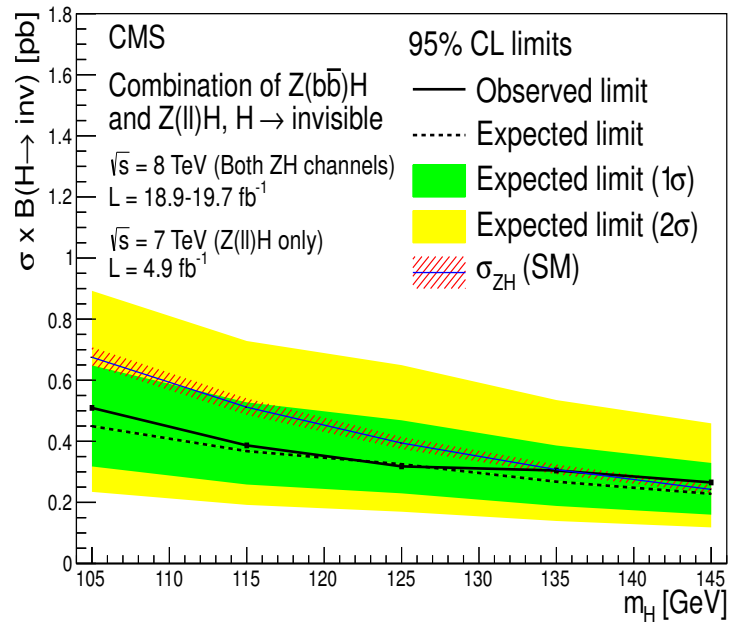

Figure 4: Combination of CMS mono- $Z(\rightarrow \ell \ell) H$ and mono- $Z(\rightarrow b \bar{b}) H$ searches [7]. Upper limits on the production cross section of the Higgs boson times its invisible branching fraction as function of the assumed mass of the Higgs boson.

The event selection is designed to categorize $\mathrm{V}$ candidates into six signal regions, depending on whether events include 2 or 3 jets, and 0,1 , or $2 b$-tags. The selection cuts are optimized in four regions of $E_{\mathrm{T}}^{\text {miss }}$. The two leading jets are required to be close to each other but to be well separated from the $E_{\mathrm{T}}^{\text {miss }}$, and to have an invariant mass $\left(\mathrm{m}_{j j}\right)$ consistent with the $\mathrm{V}$ mass.

The main backgrounds are the $\mathrm{V}$ plus jets and $t \bar{t}$ productions. They are determined via a likelihood fit to the $E_{\mathrm{T}}^{\text {miss }}$ distribution in the signal regions and the sideband regions, with the latter defined by events failing the cut on $\mathrm{m}_{j j}$. The likelihood fit is also performed to the $p_{\mathrm{T}}$ distribution of the $W$, the $Z$, and the $e-\mu$ system in enriched $W, Z$, and $t \bar{t}$ regions, respectively.

Upper bounds on $\sigma \times \mathrm{BR}(H \rightarrow$ invisible $)$ are extracted form the combined likelihood fit in the signal, control, and sideband regions. Results with different assumed $\mathrm{m}_{H}$ are presented in Figure 5. In the case of a SM Higgs boson at a mass of $125 \mathrm{GeV}$, the observed and expected upper limits on $\mathrm{BR}(H \rightarrow$ invisible $)$ amount to 0.78 and 0.86 , respectively.

\section{Search with the vector boson fusion signature}

Invisible decays of a Higgs boson produced via VBF have been investigated by both the ATLAS and CMS experiments, using datasets at $\sqrt{s}=8 \mathrm{TeV}$ totalizing $20 \mathrm{fb}^{-1}$ of integrated luminosity $[9,10]$.

Both analyses are concentrating on events with two high- $p_{\mathrm{T}}$ jets in opposite hemispheres that are well separated in pseudo-rapidity, and with a large $\mathrm{m}_{j j}$. The main background originates from the production of the $Z$ boson in association with jets, followed by its decay to neutrinos. CMS determines it from a control region consisting of $Z$ bosons decaying to muons. By contrast, ATLAS considers both $Z$ and $W$ leptonic decays as control regions, and performs a likelihood fit to the $E_{\mathrm{T}}^{\text {miss }}$ using both the control and signal regions. 
Under the hypothesis of a SM Higgs boson with $\mathrm{m}_{H}=125 \mathrm{GeV}$, the observed (expected) upper bounds on $\mathrm{BR}(H \rightarrow$ invisible $)$ are equal to $0.57(0.40)$ and $0.28(0.31)$, as derived by CMS and ATLAS, respectively. Figure 6 provides the CMS upper limits on $\sigma \times \mathrm{BR}(H \rightarrow$ invisible $)$ for different assumed $\mathrm{m}_{H}$. The less sensitive results from CMS are due to a larger uncertainty on the estimate of the electroweak background. This originates from the limited statistics that is specific to the use of a single $Z$ control region when estimating the main background. The expected CMS bound would improve to 0.33 if the $Z$ to muons control region would have as much statistics as $W$ to muon decays. For both experiments, the VBF channel nevertheless proves to be the most sensitive to constrain invisible decays of the Higgs boson.

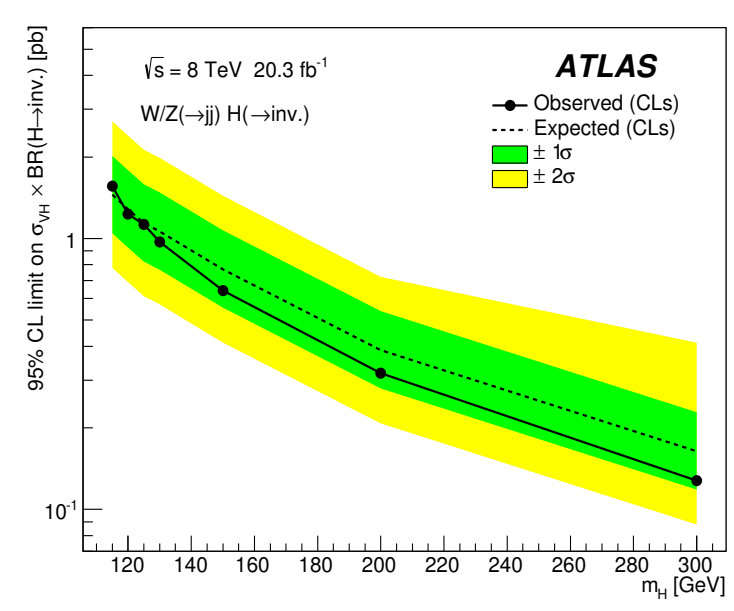

Figure 5: ATLAS $W / Z(\rightarrow j j) H$ search [8]. Upper limits on the production cross section of the Higgs boson times its invisible branching fraction as function of the assumed mass of the Higgs boson.

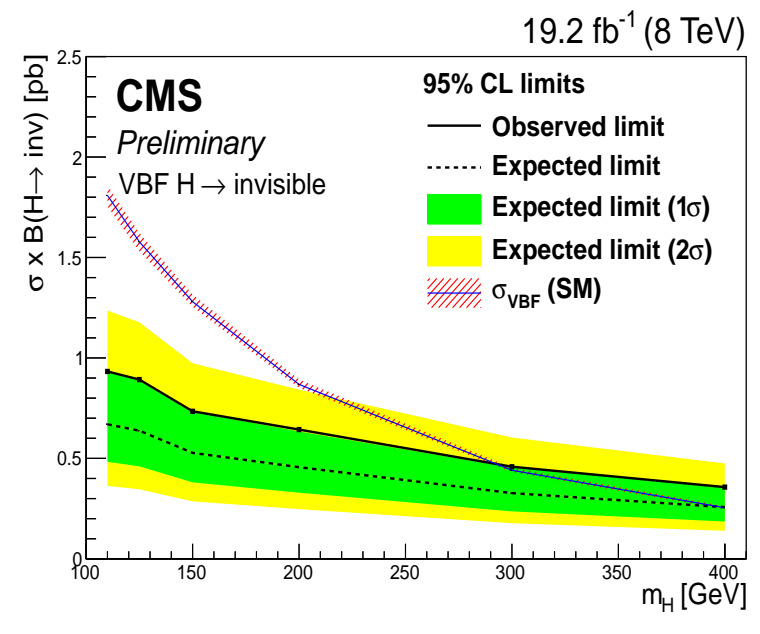

Figure 6: CMS VBF search [10]. Upper bounds on the production cross section of the Higgs boson times its invisible branching fraction as function of the assumed mass of the Higgs boson.

\section{Dark Portal}

The Higgs-portal models provide renormalizable coupling between the SM Higgs boson and the hidden sector. In particular, these theories allow to reinterpret the upper limits on $\mathrm{BR}(H \rightarrow$ invisible) into upper bounds on the spin-independent DM-Nucleon scattering cross section [1,2]. It is thus possible to compare the obtained results to those of the astroparticle experiments performing direct DM searches. Such comparisons from CMS and ATLAS are provided in Figures 7 and 8 , as function of the mass of the DM candidate $\left(\mathrm{m}_{\mathrm{DM}}\right)$. The reinterpretations are computed assuming scalar, vector, or fermionic interactions between the Higgs boson and DM. The CMS bounds in Figure 7 only include the analyses of Reference [7], and thus do not account for the most sensitive channel, namely the search in the VBF channel. To the contrary, the ATLAS results from Figure 8 only includes the VBF channel [9]. Both figures illustrate the absence of sensitivity if $\mathrm{m}_{\mathrm{DM}}$ is larger than half of $\mathrm{m}_{H}$, while showing especially sensitive limits at low $\mathrm{m}_{\mathrm{DM}}$. 


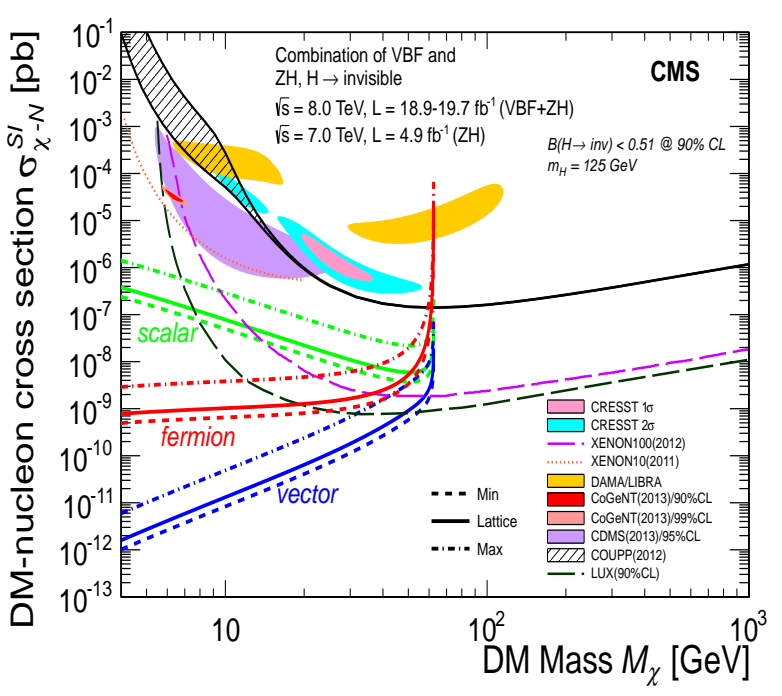

Figure 7: CMS Higgs-portal results [7]. Upper bounds on the DM-nucleon spin-independent cross section as function of the mass $M_{\chi}$ of the DM candidate. Results from CMS at the $90 \%$ confidence level are compared to astroparticle experiments.

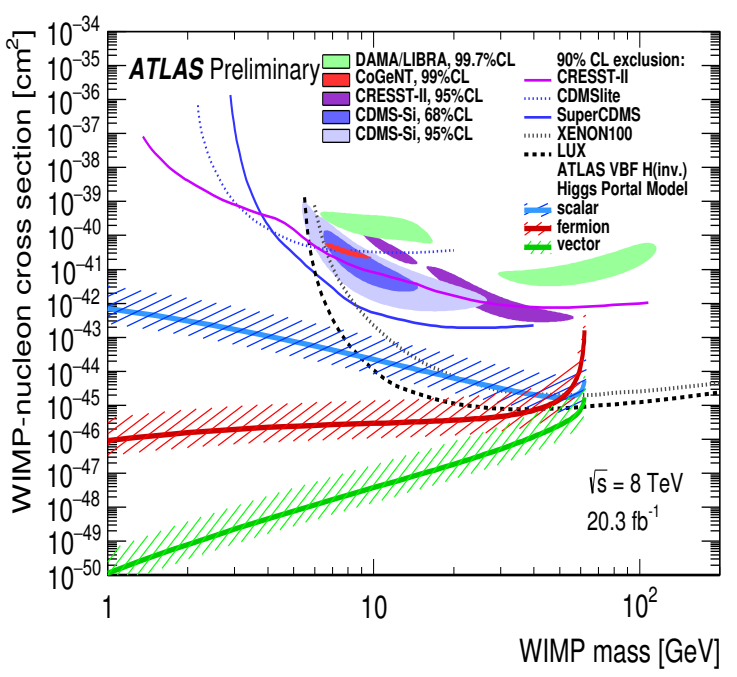

Figure 8: ATLAS Higgs-portal results [11]. Upper limits on the DM-nucleon spin-independent cross section as function of the mass of the DM candidate, referred to as a weakly interacting massive particle (WIMP). Results from ATLAS at the 90\% confidence level are compared to astroparticle experiments.

\section{Conclusion}

Searches for invisible decays of the Higgs boson have been reported by both the ATLAS and CMS Collaborations, using up to $20 \mathrm{fb}^{-1}$ of data collected from proton-proton collisions at $\sqrt{s}=7$ or $8 \mathrm{TeV}$. Various final states have been scrutinized and the expectation from the SM, determined with accuracy, proved to be in agreement with the observed data. As a consequence, stringent limits on the Higgs boson production cross section times its invisible branching fraction have been set. The interpretation of these bounds in the context of Higgs-portal models provided constraints on the production of DM candidates, and showed complementarity to astroparticle experiments focusing on the direct detection of DM.

\section{References}

[1] S. Kanemura et al, Phys. Rev. D 82, 055026 (2010) [hep-ph/1005.5651].

[2] A. Djouadi et al, Phys. Lett. B 709, 65 (2012) [hep-ph/1112.3299].

[3] ATLAS Collaboration, Eur. Phys. J. C 75, 299 (2015) [hep-ex/1502.01518v2].

[4] CMS Collaboration, CMS-PAS-EXO-12-055 (2015).

[5] CMS Collaboration, CMS-PAS-JME-09-001 (2009).

[6] ATLAS Collaboration, Phys. Rev. Lett. 112, 201802 (2014) [hep-ex/1402.3244].

[7] CMS Collaboration, Eur. Phys. J. C 74, 2980 (2014) [hep-ex/1404.1344]. 
[8] ATLAS Collaboration, Eur. Phys. J. C 75, 337 (2015) [hep-ex/1504.04324].

[9] ATLAS Collaboration, CERN-PH-EP-2015-186 (2015) [hep-ex/1508.07869].

[10] CMS Collaboration, CMS-PAS-HIG-14-038 (2015).

[11] ATLAS Collaboration, CERN-PH-EP-2015-191 (2015) [hep-ex/1509.00672v1]. 